\title{
Correlations between total cell concentration, total adenosine tri-phosphate concentration and heterotrophic plate counts during microbial monitoring of drinking water
}

\author{
E. Siebel ${ }^{1}$, Y. Wang ${ }^{1,2}$, T. Egli ${ }^{1,2}$, and F. Hammes ${ }^{1}$ \\ ${ }^{1}$ Swiss Federal Inst. of Aquatic Sci. and Technol. (Eawag), Überlandstr. 133, 8600 Dübendorf, Switzerland \\ ${ }^{2}$ Institute of Biogeochemistry and Pollutant Dynamics, ETH Zürich, 8092 Zürich, Switzerland \\ Received: 31 January 2008 - Published in Drink. Water Eng. Sci. Discuss.: 11 February 2008 \\ Revised: 19 May 2008 - Accepted: 19 May 2008 - Published: 2 June 2008
}

\begin{abstract}
The general microbial quality of drinking water is normally monitored by heterotrophic plate counts (HPC). This method has been used for more than 100 years and is recommended in drinking water guidelines. However, the HPC method is handicapped because it is time-consuming and restricted to culturable bacteria. Recently, rapid and accurate detection methods have emerged, such as adenosine tri-phosphate (ATP) measurements to assess microbial activity in drinking water, and flow cytometry (FCM) to determine the total cell concentration (TCC). It is necessary and important for drinking water quality control to understand the relationships among the conventional and new methods. In the current study, all three methods were applied to 200 drinking water samples obtained from two local buildings connected to the same distribution system. Samples were taken both on normal working days and weekends, and the correlations between the different microbiological parameters were determined. TCC in the samples ranged from $0.37-5.61 \times 10^{5} \mathrm{cells} / \mathrm{ml}$, and two clusters, the so-called high (HNA) and low (LNA) nucleic acid bacterial groups, were clearly distinguished. The results showed that the rapid determination methods (i.e., FCM and ATP) correlated well $\left(R^{2}=0.69\right)$, but only a weak correlation $\left(R^{2}=0.31\right)$ was observed between the rapid methods and conventional HPC data. With respect to drinking water monitoring, both FCM and ATP measurements were confirmed to be useful and complimentary parameters for rapid assessing of drinking water microbial quality.
\end{abstract}

\section{Introduction}

For more than 100 years, microbial characterization of drinking water has relied on heterotrophic plate counts (HPC) (Sartory, 2004). This method is used world-wide for monitoring drinking water quality in the distribution network and for assessing the efficacy of treatment steps (Allen et al., 2004; Reasoner and Geldreich, 1985). In Switzerland the statutory limit of HPC bacteria has been set to 20 colony forming units per $\mathrm{ml}(\mathrm{CFU} / \mathrm{ml})$ after treatment, and to $300 \mathrm{CFU} / \mathrm{ml}$ within the distribution system $(\mathrm{HyV}$, 2006; SLMB, 2000), which concurs with world-wide accepted guidelines (WHO, 2006). Despite the common us-

Correspondence to: F. Hammes

(frederik.hammes@eawag.ch) age of HPC, it is reported that HPC concentrations can vary widely in drinking water distribution networks (Allen et al., 2004; Pepper et al., 2004). It has been discussed previously that HPC results are influenced by various factors such as cultivation media, incubation temperature and the selective culturability of natural bacteria (Hammes et al., 2008; Sartory, 2004). Moreover, the HPC method is time-consuming, requiring usually 3-7 days before results are available. There is thus a niche for the development of rapid, easy and accurate methods to quantify bacteria in drinking water.

The development of fluorescent staining methods facilitated the accurate measurement of the total cell concentration (TCC) with epi-fluorescence microscopy, laser-scanning microscopy and flow cytometry (Rinta-Kanto et al., 2004; Hammes et al., 2008). The ability to enumerate the complete bacterial cell concentration has revealed a huge discrepancy between concentrations of cells detected with

Published by Copernicus Publications on behalf of the Delft University of Technology. 


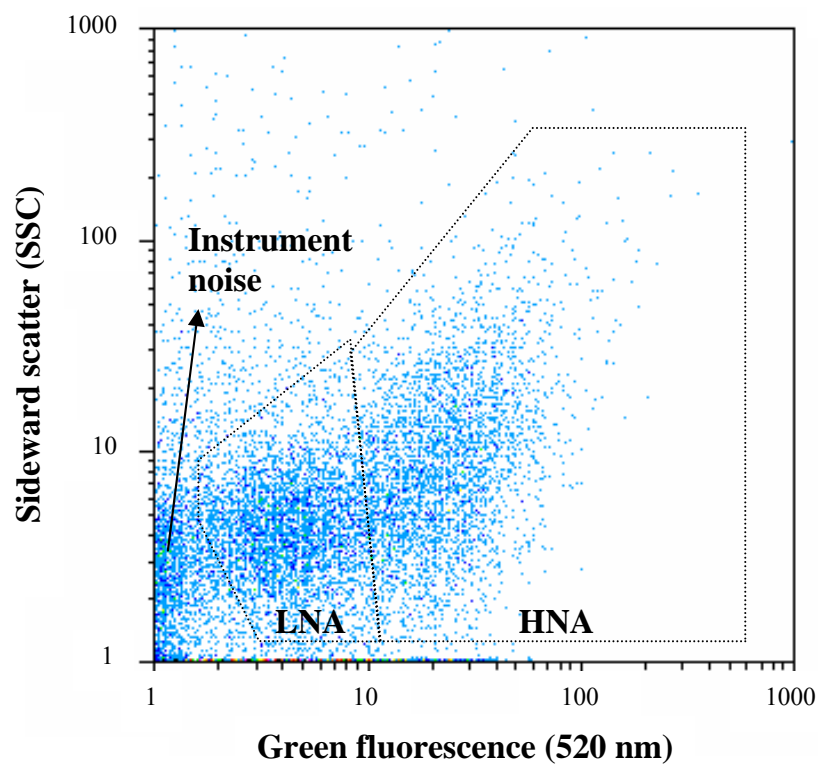

Figure 1. A 2-dimensional flow cytometry dot-plot of green fluorescence $(520 \mathrm{~nm})$ and sideward scattered light (SSC), distinguishing so-called high nucleic acid (HNA) and low nucleic acid (LNA) content bacteria.

conventional HPC and with TCC methods in aquatic environments (Hammes et al., 2008). TCC measurement with flow cytometry has clear benefits: it is fast, highly reproducible and it detects all bacteria irrespective of their culturability. We have also previously demonstrated the value of this parameter when describing microbial events that occur during drinking water treatment (Hammes et al., 2008). An alternative method gaining prominence is adenosine tri-phosphate (ATP) measurements. It is a rapid and easy method for the detection of viable bacteria in drinking water (Hammes et al., 2008) and has previously been correlated to conventional HPC data (Deininger and Lee, 2001; Delahaye et al., 2003). However, ATP is not yet widely used in the drinking water industry, due to a lack of knowledge on average ATP concentrations in natural bacteria. There is a need to understand ATP measurements and to correlate ATP data to biomass. For new methods to gain acceptance amongst practitioners, it is essential to understand the relationship (if there is any) among the various methods.

In this study, flow cytometric TCC measurements were compared with total ATP measurements and HPC data on tap water that does not contain disinfectant residuals. All three methods were applied on a local drinking water distribution system in two separate office buildings during a normal working day and a weekend day. Their correlations were determined and evaluated.

\section{Materials and methods}

\subsection{Sampling}

Drinking water samples were collected from two buildings at our research facility (Eawag, Dübendorf, Switzerland). Sampling from both buildings took place on two separate days, one working day (Wednesday) and one weekend day (Sunday) to observe the effect of regular and non-regular usage. Samples were taken from 8 a.m. to 5 p.m. at one hour intervals and at 8 a.m. on the following morning to account for temporal changes during a day. Tap water from 10 separate floors in both buildings was sampled directly into sterile $15 \mathrm{ml}$ Falcon tubes without any prior water flushing. Samples were processed directly after sampling.

\subsection{Flow cytometric total cell counts measurements}

To measure the total cell concentration (TCC), $900 \mu \mathrm{l}$ of the water sample was stained with $9 \mu$ l of SYBR ${ }^{\circledR}$ Green I nucleic acid stain (1:100 diluted in filtered DMSO; Invitrogen $^{\mathrm{TM}}$, Oregon, USA) and incubated in the dark for $10 \mathrm{~min}$ before measuring with a Partec CyFlow ${ }^{\circledR}$ Space flow cytometer (Partec GmbH, Münster, Germany). Flow cytometric measurements were performed as described previously (Hammes et al., 2008). The Partec CyFlow ${ }^{\circledR}$ Space is equipped with a volumetric counting hardware with a measured quantification limit of $<1000$ cells/ml (Hammes et al., 2008). TCC was also determined separately for two specific FCM clusters, namely the so-called low (LNA) and high (HNA) nucleic acid content bacteria, as described by Lebaron et al. (2001) and Gasol et al. (1999) (see also Fig. 1).

\subsection{Total ATP concentration}

Total ATP concentration was determined as described in Berney et al. (2006) using the Promega Bac Titer-Glo ${ }^{\mathrm{TM}} \mathrm{Mi}-$ crobial Cell Viability Assay (Promega Corporation, Madison, WI, USA). All samples were measured in triplicate. A standard curve with dilutions of a known rATP standard (Promega Corporation) was completed before the experiments and the total ATP concentration was calculated based on the standard curve. Free ATP was measured in selected samples by filtering the samples through $0.1 \mu \mathrm{m}$ sterile filters (Millipore, USA). ATP-per-cell was calculated from total ATP measurements and flow cytometry measurements as follows:

ATP-per-cell $(\mathrm{g} /$ cell $)=\frac{\text { total ATP concentration }(\mathrm{g} / \mathrm{L})}{\text { cell concentration }(\text { cells } / \mathrm{L})}$ 


\subsection{Heterotrophic plate counts (HPC)}

HPC was performed according to the Swiss guidelines for drinking water (SLMB, 2000). In short, PCA agar plates (BBL Standard Methods Agar, MD, USA) were prepared following instructions provided by the manufacturer. Serial dilutions of the water samples were done in decimal steps in sterile $0.9 \% \mathrm{NaCl}$ solution. $100 \mu \mathrm{l}$ of the sample were spreadplated from two dilutions $\left(10^{0}\right.$ and $\left.10^{-1}\right)$, each in triplicate, to assess methodological variation. The plates were incubated at $30^{\circ} \mathrm{C}$ for $72 \mathrm{~h}$. Colony forming units (CFU) were counted with an automatic plate reader (aCOLyte, SYNBIOSIS, Cambridge, UK).

\section{Results and discussion}

\subsection{Overview of the measurements with different methods}

Flow cytometric (FCM) measurements have been used effectively before to characterize bacteria in drinking water (Hoefel et al., 2003; Hammes et al., 2008). In our study, a total of 200 samples were collected and analyzed from two buildings over four separate days. For all data below, the range, average and standard deviation $(n=200)$ are given. Total cell concentrations (TCC) from the drinking water samples varied in the range of $0.37-5.61 \times 10^{5}$ cells $/ \mathrm{ml}$. The average TCC was $1.49( \pm 0.91) \times 10^{5}$ cells $/ \mathrm{ml}$, which concurs with previous data reported for Swiss drinking water (Hammes et al., 2008). In general, if a tap was used frequently, TCC numbers were low, but slight changes in bacterial concentrations could still be detected. In all samples analyzed, two specific bacterial clusters were observed on two-dimensional FCM dot-plots, distinguished by size (as measured with sideward scattered (SSC) light) and green fluorescence intensity $(520 \mathrm{~nm})$ (Fig. 1). These two clusters represent the so-called low (LNA) and high (HNA) nucleic acid content bacteria, which have been commonly observed in seawater and freshwater with flow cytometry (Gasol et al., 1999; Lebaron et al., 2001). The concentrations of LNA and HNA bacteria in each sample were also counted separately. HNA bacteria, which previously have been related to microbial activity (Lebaron et al., 2001), represented usually about $60 \%$ of the TCC (data not shown) and throughout the study the HNA/LNA ratio remained constant, irrespective of the tap location, sampling time or TCC variation. The standard deviation on triplicate FCM measurements was always below 5\%, which is similar to former studies and underlines the high reproducibility of flow cytometric TCC measurements (Hammes et al., 2008; Hammes and Egli, 2005).

An alternative method for the characterization of bacteria in drinking water is adenosine tri-phosphate (ATP) measurements (Deininger and Lee, 2001; Delahaye et al., 2003; Hammes et al., 2008). This method is rapid and easy to perform and its instrumentation is rather affordable. The total
ATP concentration for all samples was in the range of 0.005$0.094 \mathrm{nM}$ ATP and the average ATP concentration was 0.023 $( \pm 0.017) \mathrm{nM}$ ATP. Based on the TCC from flow cytometric measurements, the average cellular ATP content was calculated (Eq. 1) and the results varied from $0.05-2.09 \times 10^{-16} \mathrm{~g}$ ATP/cell with an average of $6.87( \pm 1.42) \times 10^{-17} \mathrm{~g} \mathrm{ATP} /$ cell, which is comparable to previously reported values for natural communities in drinking water (Frundzhyan and Ugarova, 2007; Hammes et al., 2008; Velten et al., 2007). The advantage of having an average ATP-per-cell value is that it can be used for converting ATP data to bacterial concentrations. However, it is noted that the value reported in this study makes the broad assumption that all the cells detected with FCM were alive and containing a similar ATP concentration. Differences in species, physiological state and cell size can all affect the ATP-per-cell concentrations. With respect to reproducibility, the average standard deviation of all the ATP data was $13 \%$, which is close to deviation values in other studies (Hammes et al., 2008; Velten et al., 2007). The free ATP concentration was always below $5 \%$ in the selected samples that were tested, and thus not considered an issue in this case. However, free ATP can have a large impact on ATP measurements, and should be considered when different water samples are analysed (Hammes et al., 2008).

Conventional heterotrophic plate counts (HPC) results were on average two orders of magnitude lower than the TCC from flow cytometric measurements. No more than $8.6 \%$ (average $1.6 \%$ ) of the TCC were detected with HPC. The average concentration of HPC was $2.28 \pm 0.317 \times 10^{3} \mathrm{CFU} / \mathrm{ml}$. Heterotrophic bacteria usually form a fraction below $1 \%$ of total cell counts (van der Kooij, 2003; Hammes et al., 2008). Based on this value and referring to the Swiss guidelines of $300 \mathrm{CFU} / \mathrm{ml}$ for drinking water (HyV, 2006; SLMB, 2000), the obtained values were about 10-times higher in this particular distribution system than expected. The finished water from the treatment plant that supplies this system has a typical HPC concentration of $<20 \mathrm{CFU} / \mathrm{ml}$ (unpublished data). The reason for such an increase might be regrowth; both planktonic and biofilm bacteria within the building's distribution system and also individual taps might affect the water quality (Pepper et al., 2004; van der Kooij, 2003). The average standard deviation of $14 \%$ is reasonable, but considerably higher compared to a constant error below 5\% in flow cytometric TCC measurements.

It should be considered that these measurements were all done on water originating from the same treatment plant and the same distribution network (with separation on building level), and therefore should not necessarily be seen as representative of all water types and conditions. For example, chlorinated water samples might contain dead cells that are still intact (thus measured with TCC), but without ATP. In addition, cell volume certainly has an influence on cellular ATP content. Therefore, the combination of flow cytometry with viability staining (Hoefel et al., 2003), as well as FCM biomass/biovolume estimation (Felip et al., 


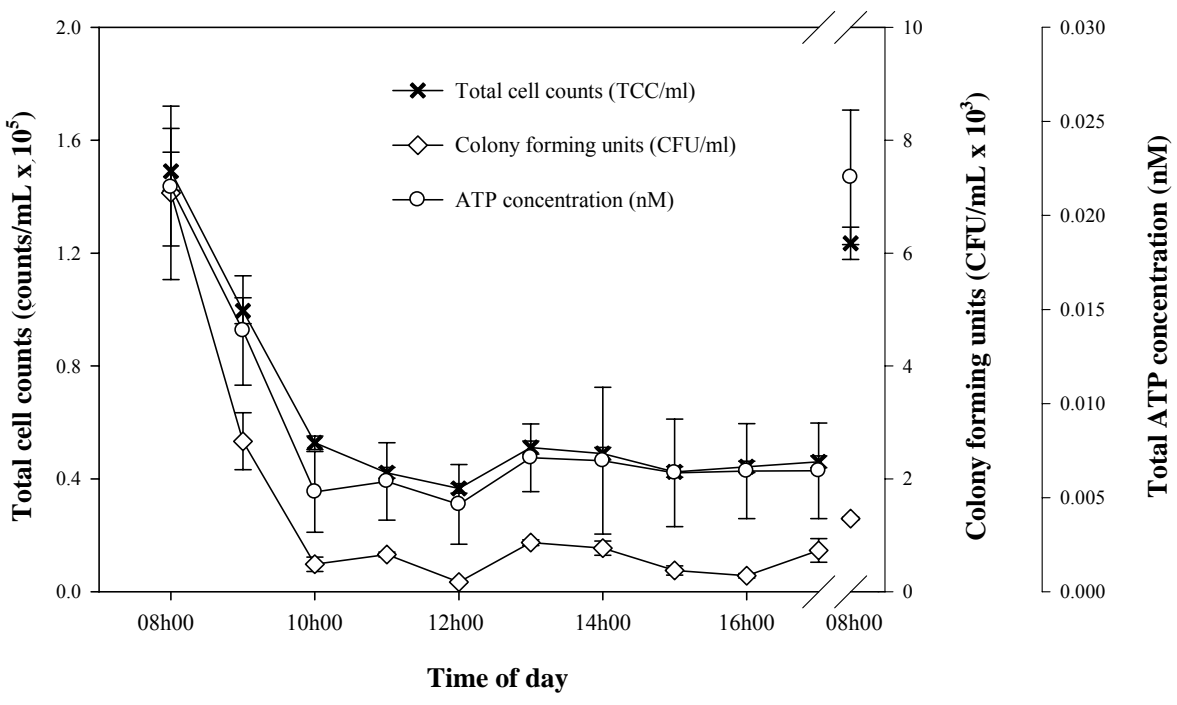

Figure 2. Daily changes of total cell counts (TCC), colony forming units (CFU) and total adenosine tri-phosphate (ATP) concentration for one representative drinking water tap, sampled on a normal working day. Error bars indicate the standard deviation on triplicate measurements.

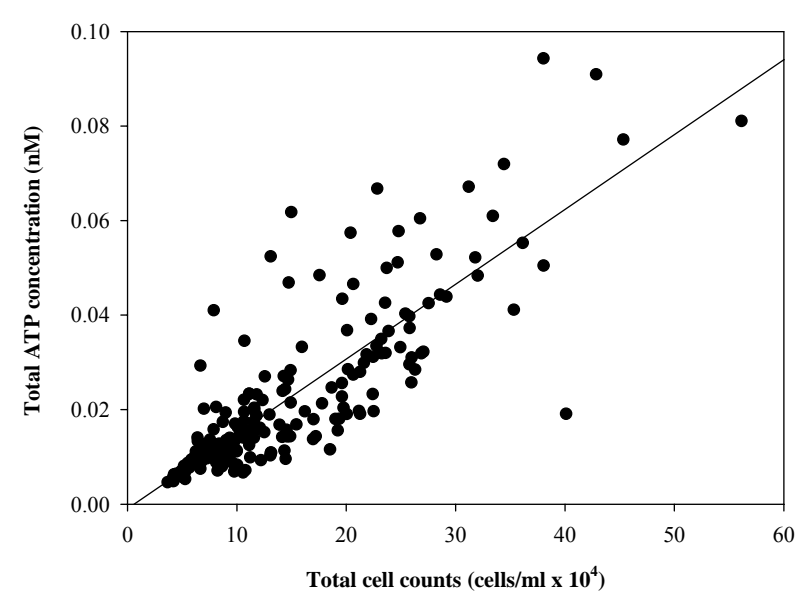

Figure 3. Correlation between total ATP concentrations and total cell concentrations of all samples $(n=200)$. All data points represent average values of triplicate measurements.

2007), can be considered as further alternatives to improve these rapid methods (specifically in relation to ATP measurements). Nonetheless, the advantage of sampling the same distribution network on a large scale is that the methods can be assessed without the influences of factors such as source water or disinfectant residuals.

\subsection{Data from one representative tap}

The changes and relationships among all three parameters from one representative tap of Building 1 during a normal working day are shown in Fig. 2. A rapid decrease in TCC and HPC along with the total ATP concentration was ob- served. It is evident that all three methods rendered the highest values at 8 a.m., which is attributed to regrowth during overnight stagnation in the tap. All values decreased until 10 a.m., linked to regular use of the taps before stabilizing during the day and again increasing overnight. In this example, the HPC did not increase as much overnight as the other parameters. All three methods from this specific tap showed a very similar pattern and significant correlations as well. The best correlation was between the TCC and ATP concentrations $\left(R^{2}=0.96 ; n=11\right)$, while correlations between HPC and TCC $\left(R^{2}=0.72\right)$ and HPC and ATP $\left(R^{2}=0.56\right)$ were also recorded. These results showed that both rapid methods (TCC and ATP) can detect the similar pattern as the conventional HPC method, but with the clear advantage of being fast, accurate and reproducible. Additionally, we observed that small changes in HPC data were also detected by flow cytometry even though there were two orders of magnitude difference in the actual values (Fig. 2). However, it should be pointed out that Fig. 2 exemplifies an ideal course of data points. Results from other floors had often more variations in daily changes, and amongst the various parameters.

\subsection{Overall correlations among the three different param- eters}

Despite of the above-mentioned differences between the different buildings, floors and sampling days, the overall TCC and ATP data correlated very well with each other $\left(R^{2}=0.69\right)$ and had a high statistical significance $(p \ll 0.001 ; n=200)$ when all data were considered (Fig. 3). However, there is also an apparent difference between the TCC and ATP measurements. This may lead to a general question such as: why 
Table 1. Linear regression analysis of different measurement parameters. $R^{2}$-values were calculated from different buildings, on different days and all data summarized $(n=200)$.

\begin{tabular}{lcccc}
\hline & TCC vs. ATP & TCC vs. HPC & HPC vs. ATP & HNA vs. ATP \\
\hline Building 1 & 0.71 & 0.25 & 0.31 & 0.76 \\
Building 2 & 0.63 & 0.45 & 0.29 & 0.56 \\
\hline Wednesday & 0.66 & 0.34 & 0.17 & 0.63 \\
Sunday & 0.63 & 0.44 & 0.29 & 0.60 \\
\hline all data & 0.69 & 0.36 & 0.31 & 0.67 \\
\hline
\end{tabular}

would ATP correlate or not correlate with TCC? Most likely, ATP would correlate well with TCC if most of the cells are alive and contain similar amounts of ATP, expecting a correlation coefficient close to 1 . Evidently this is not the case and can be explained with various reasons. The uncertainty of ATP measurements $\left(R^{2}=0.69 \neq 1\right)$ towards TCC is probably not related to the error of either method, but occurs because different bacterial species and different viability states of bacterial cells could influence individual cellular ATP concentrations. In fact, in a separate study we have shown that an even better correlation is found between ATP and the fraction of esterase-positive (CFDA-stained) cells in drinking water ${ }^{1}$. Nevertheless, the combination of the TCC and ATP methods gives a complementary overall picture of the microbial quality in the drinking water distribution system.

Furthermore, we have also observed a similar overall correlation between HNA bacteria and ATP $\left(R^{2}=0.67\right)$ (Table 1). It has been suggested that the HNA fraction gives a representation of the active bacterial population in a water sample (Gasol et al., 1999; Lebaron et al., 2001). The fact that the correlation with ATP has not improved when compared to HNA (opposed to TCC), contradicts this opinion to some extent, and suggests that a mere HNA/LNA flow cytometric separation might be inadequate to interpret bacterial viability and activity in a drinking water sample. In addition, the consistent presence of LNA bacteria at high cell concentrations ( $40 \%$ of TCC) in the drinking water distribution system, indicates that this group of bacteria may also contribute to the bacterial activity.

In comparison to the strong correlation between TCC and ATP, correlation coefficients between HPC vs. ATP $\left(R^{2}=0.31\right)$ and HPC vs. TCC $\left(R^{2}=0.36\right)$ were considerably lower, also when the data were separated to track possible distinctive changes day-wise and/or building-wise (Table 1). The correlation between ATP and HPC $\left(R^{2}=0.31\right.$; Fig. 4) is similar to that reported by Delahaye et al. (2003) even though different cultivation methods were used in the latter study. Both the studies of Delahaye et al. (2003) and Deininger and Lee (2001) concluded that rapid ATP measurement is a

\footnotetext{
${ }^{1}$ Berney et al., unpublished data
}

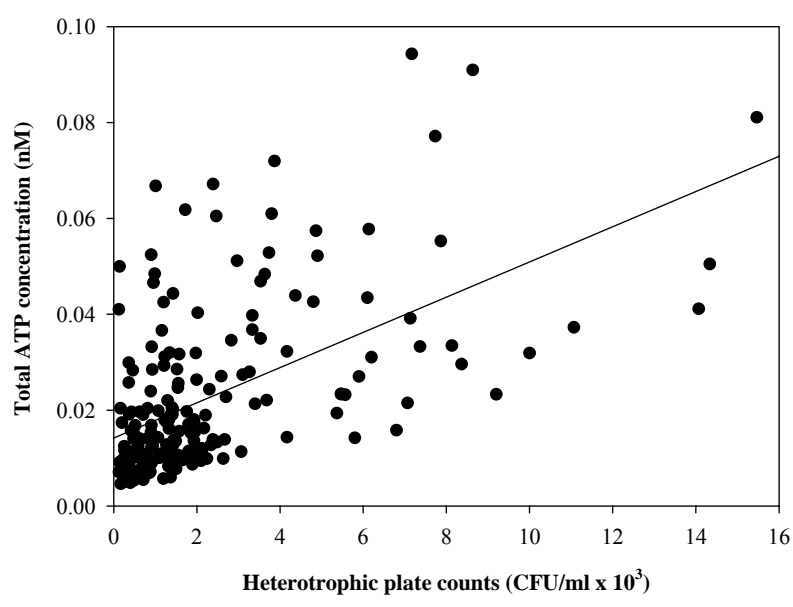

Figure 4. Correlation between total ATP concentrations heterotrophic plate counts (HPC) of all samples $(n=200)$. All data points represent average values of triplicate measurements.

valuable drinking water parameter, based on the correlation thereof to conventional HPC data. Though our study comes to the same conclusion about ATP measurements, the data presented in Figs. 3 and 4 and Table 1 clearly suggest that ATP correlates more accurately to TCC than to HPC. We believe that this is a direct result of the selective nature of the HPC method, where only a small percentage of the viable (and thus ATP-containing) bacteria in a water sample can be cultivated on synthetic media under selective laboratorydefined conditions (Hammes et al., 2008).

\section{Conclusions}

Rapid enumeration methods are crucial for drinking water quality monitoring. Flow cytometry and total ATP measurements are both sensitive and rapid methods that can be performed easily without the handicap of a selective trait such as culturability. In comparison, the enumeration of culturable heterotrophic bacteria is obviously more time and labour consuming. We have found that there is a strong correlation between TCC and ATP data. Therefore, application of both 
methods simultaneously forms a powerful combination of rapid and accurate microbial characterization of drinking water. It is clear that both methods, similar to HPC, are not direct indicators for hygienic quality, but should rather be used for the assessment of the general quality of drinking water and biological stability of drinking water. Ongoing assessments of different water types and distribution conditions, in comparison with conventional HPC measurements, could provide the data with which to challenge existing guidelines and regulations.

Acknowledgements. The authors kindly acknowledge financial assistance from the Eawag Wave21 project and the 6th EU framework project TECHNEAU (018320).

Edited by: H. van Dijk

\section{References}

Allen, M. J., Edberg, S. C., and Reasoner, D. J.: Heterotrophic plate count bacteria - what is their significance in drinking water?, Int. J. Food Microbiol., 92(3), 265-274, 2004.

Berney, M., Weilenmann, H. U., and Egli, T.: Flow-cytometric study of vital cellular functions in Escherichia coli during solar disinfection (SODIS), Microbiol.-SGM, 152, 1719-1729, 2006.

Deininger, R. A. and Lee, J.: Rapid determination of bacteria in drinking water using an ATP assay, Field Anal. Chem. Tech., 5(4), 185-189, 2001.

Delahaye, E., Welte, B., Levi, Y., Leblon, G., and Montiel, A.: An ATP-based method for monitoring the microbiological drinking water quality in a distribution network, Water Res., 37(15), 3689-3696, 2003.

Felip, M., Andreatta, S., Sommaruga, R., Straskrábová, V., and Catalan, J.: Suitability of flow cytometry for estimating bacterial biovolume in natural plankton samples: comparison with microscopy data, Appl. Environ. Microbiol., 73, 4508-4514, 2007.

Frundzhyan, V. and Ugarova, N.: Bioluminescent assay for total bacterial contamination of drinking water, Luminescence, 22, 241-244, 2007.

Gasol, J. M., Zweifel, U. L., Peters, F., Fuhrman, J. A., and Hagstrom, A.: Significance of size and nucleic acid content heterogeneity as measured by flow cytometry in natural planktonic bacteria, Appl. Environ. Microb., 65(10), 4475-4483, 1999.
Hammes, F., Berney, M., Wang, Y., Vital, M., Köster, O., and Egli, T.: Flow-cytometric total bacterial cell counts as a descriptive microbiological parameter for drinking water treatment processes, Water Res., 42(1-2), 269-277, 2008.

Hammes, F. A. and Egli, T.: New method for assimilable organic carbon determination using flow-cytometric enumeration and a natural microbial consortium as inoculum, Environ. Sci. Technol., 39(9), 3289-3294, 2005.

Hoefel, D., Grooby, W. L., Monis, P. T., Andrews, S., and Saint, C. P.: Enumeration of water-borne bacteria using viability assays and flow cytometry: a comparison to culture-based techniques, J. Microbiol. Meth., 55, 585-597, 2003.

HyV: Hygieneverordnung (Hygiene regulation) des EDI (Eidgenössische Departement des Innern), Anhang 2, 2005.

Lebaron, P., Servais, P., Agogue, H., Courties, C., and Joux, F.: Does the high nucleic acid content of individual bacterial cells allow us to discriminate between active cells and inactive cells in aquatic systems?, Appl. Environ. Microb., 67(4), 1775-1782, 2001.

Pepper, I. L., Rusin, P., Quintanar, D. R., Haney, C., Josephson, K. L., and Gerba, C. P.: Tracking the concentration of heterotrophic plate count bacteria from the source to the consumer's tap, Int. J. Food Microbiol., 92(3), 289-295, 2004.

Reasoner, D. J. and Geldreich, E. E.: A new medium for the enumeration and subculture of bacteria from potable water, Appl. Environ. Microb., 49(1), 1-7, 1985.

Rinta-Kanto, J. M., Lehtola, M. J., Vartiainen, T., and Martikainen, P. J.: Rapid enumeration of virus-like particles in drinking water samples using SYBR green I-staining, Water Res., 38, 26142618, 2004.

Sartory, D. P.: Heterotrophic plate count monitoring of treated drinking water in the UK: a useful operational tool, Int. J. Food Microbiol., 92, 297-306, 2004.

SLMB: Schweizer Lebensmittelbuch (Swiss book of food regulation), 56, E.1., 2000.

van der Kooij, D.: Managing regrowth in drinking-water distribution systems, in: Heterotrophic plate counts and drinking-water safety, edited by: Bartram, J., Cotruvo, J., Exner, M., Fricker, C., and Glasmacher, M., London, UK, World Health Organization (WHO) by IWA Publishing, 199-232, 2003.

Velten, S., Hammes, F., Boller, M., and Egli, T.: Rapid and direct estimation of active biomass on granular activated carbon through adenosine tri-phosphate (ATP) determination, Water Res., 41, 1973-1983, 2007.

WHO: World Health Organization, Guidelines for Drinking-water Quality (electronic resource): incorporating first addendum, Vol. 1, Recommendations, $3^{\text {rd }}$ Ed., 2006. 